\title{
Psychotherapie bei schizophrenen Störungen: Kein Evidenz-, sondern ein Implementierungsproblem
}

\author{
Psychotherapy of Schizophrenia: Not a Problem of Evidence, but a Problem of \\ Implementation
}

Autoren

Institute

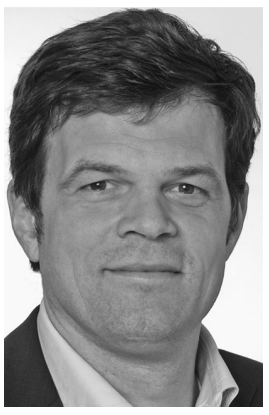

Prof. Dr. med. Andreas Bechdolf

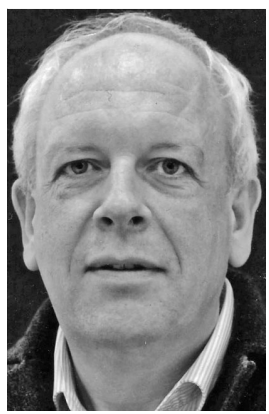

Prof. Dr. phil. Dipl.-Psych. Stefan Klingberg

\section{Bibliografie}

DOI http://dx.doi.org/

10.1055/s-0033-1359957

Psychiat Prax 2014; 41: 8-10

(c) Georg Thieme Verlag KG

Stuttgart · New York

ISSN 0303-4259

Korrespondenzadresse

Prof. Dr. med.

Andreas Bechdolf

Klinik für Psychiatrie,

Psychotherapie und

Psychosomatik, Vivantes

Klinikum am Urban

Dieffenbachstraße 1

10967 Berlin

andreas.bechdolf@vivantes.de

\author{
Andreas Bechdolf ${ }^{1,2,3}$, Stefan Klingberg ${ }^{4}$ \\ Kliniken für Psychiatrie, Psychotherapie und Psychosomatik, Vivantes Klinikum am Urban und Vivantes Klinikum \\ im Friedrichshain, Akademische Lehrkrankenhäuser Charité - Universitätsmedizin Berlin \\ ${ }^{2}$ Klinik für Psychiatrie und Psychotherapie, Klinikum der Universität zu Köln \\ ${ }^{3}$ ORYGEN Youth Health, University of Melbourne, Melbourne, Australia \\ ${ }^{4}$ Klinik für Psychiatrie und Psychotherapie, Universitätsklinikum Tübingen
}

Schizophrene Störungen gehören zu den fünf Erkrankungen, bei denen die meisten Lebensjahre in Behinderung verbracht werden [1]. Die Erkrankung geht mit großem subjektivem Leid der Betroffenen und Angehörigen einher. 5\% der Betroffenen versterben an einem Suizid [2]. Darüber hinaus erreichen nur $20 \%$ der an einer schizophrenen Störung Erkrankten eine Tätigkeit auf dem ersten Arbeitsmarkt [3], sodass die Störung auch von großer volkswirtschaftlicher Bedeutung ist. Obwohl die Pharmakotherapie die wesentliche Grundlage der Therapie der schizophrenen Störungen bildet, bleibt auch bei medikamentöser Behandlung noch eine Reihe von Problemen bestehen. Über 20\% der Patienten leiden unter persistierenden Positivsymptomen [4]. Viele Patienten zeigen auch längerfristig kognitive Störungen [5]. Trotz Medikationseinnahme besteht ein relevantes Rezidivrisiko. Viele Patienten sind nicht bereit, langfristig Antipsychotika einzunehmen und müssen fortgesetzt zur Einnahme motiviert werden [6]. Schließlich kann das Auftreten der Erkrankung selber zu bedeutsamer Selbstwertproblematik und Selbststigmatisierung führen, die eine Behandlungsbedürftigkeit für sich genommen darstellt [7].

Nachdem man jahrzehntelang davon ausging, dass psychotherapeutische Behandlung bei Patienten mit psychotischen Störungen wirkungslos sei oder gar vermieden werden sollte, konnte die Psychotherapie-Wirksamkeitsforschung in den letzten 30 Jahren zeigen, dass eine Reihe von Psychotherapiestrategien zusätzlich zur antipsychotischen Medikation eingesetzt, den Krankheitsverlauf deutlich positiv beeinflussen können. Viele der angewandten Strategien unterscheiden sich dabei nicht grundsätzlich von Psychotherapien bei anderen psychiatrischen Störungen. Da mittlerweile eine Vielzahl von Studien durchgeführt wurde, liegen jetzt verschiedene Metaanalysen zu den wesentlichen psychotherapeutischen Behandlungsstrategien bei schizophrenen
Störungen vor. Viele Therapieansätze sind auch im deutschen Versorgungssystem in klinischen Studien positiv evaluiert [8-12].

\section{Evidenzbasierte Psychotherapien $\nabla$}

Seit Mitte der 1980er-Jahre wurden Studien durchgeführt, die besonders viel Aufmerksamkeit erregt haben. Hier wurden in der Regel im Einzelsetting die kognitiven Prinzipien von A. T. Beck auf Halluzinationen und Wahn angewendet. In einer Metaanalyse von Wykes et al. [13], in die die Ergebnisse von 1964 kognitiv-verhaltenstherapeutisch behandelten Patienten (KVT) eingingen, wurde eine mittlere Effektstärke der Therapie von $\mathrm{d}=0,4$ in Bezug auf die jeweils primär untersuchte Symptomatik festgestellt. Eine Effektstärke, die in der Größenordnung des Effektes einer Clozapinumstellung bei Patienten mit persistierenden Symptomen liegt und sich durchaus mit den Psychotherapieeffekten bei depressiven Symptomen vergleichen lassen kann $[13,14]$. Den größten Effektivitätsnachweis gibt es für den rezidivprophylaktischen Effekt der sogenannten Familieninterventionen. Hier werden Information, Kommunikationstraining und Frühsymptommanagement für Angehörige und Patienten angeboten. Auch hier spricht der aktuelle Cochrane-Review von klaren Effekten ([15]; $n=721$, 14 RCTs). Sieben Patienten müssen behandelt werden, um bei einem eine Wiedererkrankung zu vermeiden (Number Needed to Treat, NNT $=7$ ). Eine Rate, die für viele rezidivprophylaktische Maßnahmen in der somatischen Medizin nicht erreicht wird [16].

Für das Trainieren der kognitiven Leistungsfähigkeit, die der medikamentösen Therapie in der Regel nur geringgradig zugänglich ist, wurde in der jüngsten Metaanalyse von McGurk et al. [17] eine der KVT der Positivsymptomatik vergleichbaren Effektstärke von 0,4 beschrieben [17]. 
Die Bearbeitung des Krankheits- und Behandlungskonzepts spielt eine zentrale Rolle in der schizophreniespezifischen Psychotherapie. Im deutschsprachigen Raum hat sich besonders der Begriff Psychoedukation für diese Behandlungsform durchgesetzt. Im aktuellen Cochrane-Review von Xia und Kollegen [18] wird festgestellt, dass jede Art von Psychoedukation Rückfall- und Wiederaufnahmeraten im Zeitraum von 9-18 Monaten nach der Intervention verringert. Neun Patienten müssen behandelt werden, um einen in der Exazerbation zu verhindern (NNT=9) [18]. In einer jüngst publizierten Metaanalyse unter Beteiligung des Erstautors konnte gezeigt werden, dass psychotherapeutische Interventionen bei Personen mit erhöhtem Psychoserisiko präventive Effekte haben und auch hier klinisch bedeutsame NNTs zwischen 4 und 8 erreicht werden können [19].

\section{Empfehlungen der Behandlungsleitlinien}

Vor dem Hintergrund der oben dargestellten Datenlage verwundert es nicht, dass die aufgelisteten psychotherapeutischen Interventionen in Behandlungsleitlinien zur schizophrenen Störung mit dem höchsten Evidenzgrad angegeben werden. Die methodisch am anspruchsvollsten gestaltete Leitlinie des National Instituts of Clinical Excellence (NICE [20]) empfiehlt, dass kognitive Therapie, Familienintervention und Frühinterventionsangebote allen Patienten mit schizophrenen Störungen angeboten werden sollten. Die NICE-Leitlinie legt sich sogar so weit spezifisch fest, dass sie empfiehlt, mindestens 16 Einzelsitzungen kognitiver Therapie für Patienten mit schizophrenen Störungen vorzuhalten (Empfehlung 8.4.10.2). Ähnliche Empfehlungen hält die in den USA vom Schizophrenia Patient Outcomes Research Team (PORT) erarbeitete Leitlinie vor [21]. Auch die für unser Versorgungsgebiet relevante Leitlinie der Deutschen Gesellschaft für Psychiatrie und Psychotherapie, Psychosomatik und Nervenheilkunde (DGPPN) von 2006 kommt zu vergleichbaren Empfehlungen [22]. Mit dem höchsten Empfehlungsgrad wird hier KVT bei medikamentös behandlungsresistenter Schizophrenie, insbesondere bei persistierenden psychotischen Symptomen, empfohlen. Ebenso wird KVT zur weiteren Reduktion des Rückfallrisikos zusätzlich zu einer adäquaten medikamentösen Therapie sowie als alleinige Therapie zur Prävention einer psychotischen Episode bei Personen mit erhöhtem Psychoserisiko empfohlen. Im Einklang mit den anderen zitierten Leitlinien wird die kognitiv-verhaltenstherapeutische Familienbetreuung als empirisch am besten abgesicherte psychosoziale Intervention zur Senkung der Wiedererkrankungsrate beschrieben.

\section{Implementierung}

$\nabla$

Wer schon einmal versucht hat, Patienten mit schizophrenen Störungen in ambulante Psychotherapie zu vermitteln, wird den Autoren zustimmen, dass abgesehen von Einzelfällen, die Vermittlungsrate katastrophal ist. Eine von der Berliner Psychotherapeutenkammer beauftragte Studie fand, dass weniger als $1 \%$ der Patienten mit schizophrenen Störungen bei niedergelassenen Psychotherapeuten in Behandlung sind [23]. 50\% sind zwar in psychiatrischer Behandlung, hier ist aber die Art der Behandlung nicht näher spezifiziert. Ähnliches findet ein Modellprojekt der Techniker Krankenkasse, die zeigte, dass Patienten mit schizophrenen Störungen weniger als $1 \%$ des Klientels niedergelassener Psychotherapeuten ausmacht [24]. Die Psychotherapie-
Richtlinie wird hier einen negativen Einfluss ausüben, da nur „Begleit-, Folge- und Residualsymptomatik“ nicht aber die Symptome der Schizophrenie selbst Gegenstand der Behandlung sein können und Psychotherapieanträge für Patienten mit schizophrenen Störungen ein höheres Ablehnungsrisiko aufweisen als Anträge für die meisten anderen Störungen. Die PsychotherapieRichtlinie steht damit derzeit in starkem Kontrast zu allen international publizierten Leitlinien und dem Stand der Forschung. Dies gilt auch für die als hocheffektive rezidivprophylaktische Maßnahme zu beschreibende Familienintervention. Als ambulante Psychotherapie kann dies derzeit nicht durchgeführt werden, weil Familienintervention kein sogenanntes Richtlinienverfahren ist, also nicht zulasten der Krankenkasse durchgeführt werden kann. Selbst für die relativ pragmatisch an das deutsche Versorgungssystem angepasste Psychoedukation fanden Rummel-Kluge und Kollegen in einer Erhebung aus dem Jahr 2000, dass nur $21 \%$ der Patienten mit schizophrenen Störungen psychoedukative Interventionen erhalten und sogar nur 2\% der Angehörigen [25]. Die niedrige Angebotsrate der für die Erreichung der positiven Psychoedukationseffekte so existenziellen Angehörigeninterventionen ist wesentlich in der mangelnden Finanzierung dieser Leistung z.B. auch im Krankenhaus begründet.

\section{Forderungen für die Praxis \\ $\nabla$}

Psychotherapeutische Interventionen bei Patienten mit schizophrenen Störungen wirken günstig auf den Symptomverlauf, auf die Rezidivprophylaxe, fördern die Medikamentencompliance und unterstützen die Krankheitsverarbeitung der Betroffenen. Darüber kann der Krankheitsverlauf wesentlich verbessert werden. Die Evidenzlage ist erschlagend.

Sicherlich ist das Wissen um die Effektivität der Psychotherapie bei Patienten mit schizophrenen Störungen noch nicht bei allen Entscheidungsträgern angekommen. Auch wird man eine noch nicht ausreichende Zahl an in diesen Strategien geschulten Therapeuten beklagen müssen. Dennoch ist das Hauptproblem klar in einer Versorgungsstruktur zu sehen, die die Anwendung von Psychotherapie bei dieser Patientengruppe erschwert.

Niedergelassene Psychotherapeuten sollten nicht durch die Psychotherapie-Richtlinie gehindert sein, sondern Anreize bekommen, Patienten mit schizophrenen Störungen zu behandeln bzw. Stundenausfall bei unzuverlässigen Patienten leichter kompensieren zu können. Interventionen mit Angehörigen sollten nicht nur ermöglicht werden, sondern auch finanziell attraktiver als Einzelsitzungen sein. Insgesamt müssen mehr Möglichkeiten geschaffen werden, schwer und akut psychisch Kranke psychotherapeutisch in unserem Gesundheitssystem zu versorgen. Zusätzlich zu mehr Anreizen, schwer Erkrankte mit komplexeren, zum teil auch Angehörige einbeziehenden Interventionen zu behandeln, würden viele Betroffene auch von einer Flexibilisierung der Sitzungszeiten profitieren. Nicht jeder Patient benötigt 40-mal 50 Minuten. 4-6 Sitzungen à 20-60 Minuten z. B. können die Medikamentencompliance und das soziale Funktionsniveau nachhaltig erhöhen [26, 27].

Im neuen Entgeltgesetz sind Modellprojekte vorgesehen. Hier besteht die Möglichkeit, psychotherapeutische Interventionen in der psychiatrischen Praxis zu stärken. Erste Erfahrungen für den Erfolg solcher Modelle gibt es schon aus Integrierten Versorgungsprojekten.

Eine Investition in Psychotherapie bei Patienten mit schizophrenen Störungen macht sich in reduzierten Wiederaufnahmeraten 
in Krankenhäusern „bezahlt“, ist also letztlich kosteneffektiv. Evidenzbasierte Psychotherapie bietet die Chance, bei vielen Betroffenen einen besseren Krankheitsverlauf zu erreichen, Behinderungen zu reduzieren und Leid bei Betroffenen und Angehörigen zu minimieren sowie gesellschaftliche Kosten zu reduzieren. Die wissenschaftliche Evidenz ist da, die Umsetzung in die klinische Praxis sollten wir jetzt angehen.

\section{Literatur}

1 Murray CJ, Lopez AD. Global mortality, disability, and the contribution of risk factors: Global Burden of Disease Study. Lancet 1997; 349: $1436-1442$

2 Hor $K$, Taylor $M$. Suicide and schizophrenia: a systematic review of rates and risk factors. J Psychopharmacol 2010; 24 (Suppl. 04): 81-90

3 Mueser KT, Salyers MP, Mueser PR. A prospective analysis of work in schizophrenia. Schizophr Bull 2001; 27: 281-296

4 Lieberman JA, Stroup TS, McEvoy JP et al. Clinical Antipsychotic Trials of Intervention Effectiveness (CATIE) Investigators. Effectiveness of antipsychotic drugs in patients with chronic schizophrenia. N Engl J Med 2005; 353: 1209-1223

5 Keefe R. Cognitive deficits in patients with schizophrenia: effects and treatment. J Clin Psychiatry 2006; 68: 8-13

6 Robinson D, Woerner MG, Alvir JM et al. Predictors of relapse following response from a first episode of schizophrenia or schizoaffective disorder. Arch Gen Psychiat 1999; 56: 241-247

7 Rüsch N, Berger M, Finzen A, Angermeyer M. Das Stigma psychischer Erkrankungen - Ursachen, Formen und therapeutische Konsequenzen. In: Berger M, Hrsg. Psychische Erkrankungen: Klinik und Therapie. 3. Auflage. München: Urban \& Fischer; 2009: 1091 - 1100

8 Bäuml J, Froböse T, Kraemer $S$ et al. Psychoeducation: a basic psychotherapeutic intervention for patients with schizophrenia and their families. Schizophr Bull 2006; 32 (Suppl. 01): S1 - S9

9 Bechdolf A, Knost B, Kuntermann $C$ et al. A randomized comparison of group cognitive-behavioural therapy and group psychoeducation in patients with schizophrenia. Acta Psychiatr Scand 2004; 110: 21 -28

10 Bechdolf A, Köhn D, Knost B et al. A randomized comparison of group cognitive-behavioural therapy and group psychoeducation in acute patients with schizophrenia: outcome at 24 months. Acta Psychiatr Scand 2005; 112: $173-179$

11 Buchkremer G, Klingberg S, Holle R et al. Psychoeducational psychotherapy for schizophrenic patients and their key relatives or care-givers: results of a 2-year follow-up. Acta Psychiatr Scand 1997; 96: 483-491

12 Klingberg S, Wittorf A, Meisner C et al. Cognitive behavioural therapy versus supportive therapy for persistent positive symptoms in psychotic disorders: The POSITIVE Study, a multicenter, prospective, singleblind, randomised controlled clinical trial. Trials 2010; 11: 123
13 Wykes T, Steel C, Everitt B et al. Cognitive behavior therapy for schizophrenia: effect sizes, clinical models, and methodological rigor. Schizophr Bull 2008; 34: 523-537

14 Driessen E, Hollon SD. Cognitive behavioral therapy for mood disorders: efficacy, moderators and mediators. Psychiatr Clin North Am 2010; 33: 537-555

15 Pharoah F, Mari J, Rathbone J et al. Family intervention for schizophrenia. Cochrane Database Syst Rev 2010; (12): CD000088

16 Psaty BM, Lumley T, Furberg CD et al. Health outcomes associated with various antihypertensive therapies used as first-line agents. JAMA 2003; 289: 2534-2544

17 McGurk SR, Twamley EW, Sitzer DI et al. A meta-analysis of cognitive remediation in schizophrenia. Am J Psychiatry 2007; 164: 1791 - 1802

18 Xia J, Merinder LB, Belgamwar MR. Psychoeducation for schizophrenia. Cochrane Database Syst Rev 2011; (06): CD002831

19 van der Gaag M, Smit F, Bechdolf A et al. Preventing a first episode of psychosis: Meta-analysis of randomized controlled prevention trials of 12 month and longer-term follow-ups. Schizophr Res 2013; 149: $56-62$

20 National Institute for Health and Clinical Excellence Schizophrenia. NICE clinical guideline 82. Core interventions in the treatment and management of schizophrenia in primary and secondary care (update). Im Internet: http://publications.nice.org.uk/schizophrenia-cg82 (Stand: 27.11.2013)

21 Dixon LB, Dickerson F, Bellack AS et al. The 2009 schizophrenia PORT psychosocial treatment recommendations and summary statements. Schizophr Bull 2010; 36: 48-70

22 Deutsche Gesellschaft für Psychiatrie und Psychotherapie, Psychosomatik und Nervenheilkunde (DGPPN). S3 Praxisleitlinien in Psychiatrie und Psychotherapie. Band 1 - Behandlungsleitlinie Schizophrenie. Darmstadt: Steinkopff-Verlag; 2005

23 Görgen W, Engler U. Ambulante psychotherapeutische Versorgung von psychosekranken Menschen sowie älteren Menschen in Berlin. Heidelberg: Verlagsgruppe Hüthig Jehle Rehm; 2005

24 Wittmann WW, Lutz W, Steffanowski A et al. Qualitätsmonitoring in der ambulanten Psychotherapie: Modellprojekt der Techniker Krankenkasse - Abschlussbericht. Hamburg: Techniker Krankenkasse; 2011

25 Rummel-Kluge C, Pitschel-Walz G, Bäuml J et al. Psychoeducation in schizophrenia - results of a survey of all psychiatric institutions in Germany, Austria, and Switzerland. Schizophr Bull 2006; 32: 765-775

26 Kemp R, Hayward P, Applewhaite G et al. Compliance therapy in psychotic patients: randomised controlled trial. BMJ 1996; 312: 345-349

27 Kemp R, Kirov G, Everitt B et al. Randomised controlled trial of compliance therapy. 18-month follow-up. Br J Psychiatry 1998; 172: 413419 